\title{
Diffraction of Laguerre-Gaussian Beam by a Helical Axicon
}

\author{
S. TOPUZOSKI* AND LJ. JANICIJEVIC \\ Institute of Physics, University Sts "Cyril And Methodius", Skopje, R. Macedonia
}

\begin{abstract}
In this article the transformation of the Laguerre-Gaussian beam with radial mode number $n$ and azimuthal mode number $l$, into a vortex, diverging or nondiverging Bessel beam with increased, decreased or zeroth phase singularity order has been investigated theoretically. We deduce and study analytical expressions for the amplitude and intensity distribution of the diffracted wave field in the process of Fresnel diffraction, using the stationary phase method, and also, for the vortex radius and the propagation distance of the output field.
\end{abstract}

PACS numbers: 42.25.Fx, 42.60.Jf

\section{Introduction}

The nondiverging ("nondiffracting") beams have attracted an increased interest due to their intriguing properties and interesting applications [1]. It was shown by Durnin et al. [2] that optical fields with Bessel function transverse amplitude profile can exhibit nondiverging properties during their propagation, remaining their transverse intensity profile unchanged in form and dimensions in a defined ranging interval. The high-order Bessel beams having the phase azimuthally varying as $\exp (\mathrm{i} l \varphi)$, where $\varphi$ is the azimuthal coordinate, $l$ is an integer, are characterised by helical phasefront and are vortex beams. In Ref. [3] the authors report about transformation of Laguerre-Gaussian beam, $L G_{n=0}^{l}$, into a Bessel beam of the same topological charge as the incident one. The possibility to transform a $L G_{n=0}^{l}$ beam into a vortex, diverging or nondiverging Bessel beam, which can have increased or decreased phase singularity order, or into a zeroth order Bessel beam, by means of a helical axicon (HA), has been shown recently in [4]. In this article we solve a more general problem than in [4] by considering the incident $L G$ beam with radial mode number different from zero, namely, the diffraction of the $L G_{n \neq 0}^{l}$ beam by helical axicon.

The incident beam is a Laguerre-Gaussian beam with radial mode number $n$ and azimuthal mode number $l>0$, defined in cylindrical coordinates as [5]

$$
\begin{gathered}
U_{n}^{l}(r, \varphi, z)=C_{l, n} \frac{w_{0}}{w(z)}\left(\frac{r \sqrt{2}}{w(z)}\right)^{l} L_{n}^{l}\left(\frac{2 r^{2}}{w^{2}(z)}\right) \\
\quad \times \exp \left(\frac{-r^{2}}{w^{2}(z)}\right) \exp \left(-\mathrm{i}\left(k z+k \frac{r^{2}}{2 R(z)}\right.\right. \\
\left.\left.-(2 n+l+1) \arctan \left(\frac{z}{z_{0}}\right)\right)\right) \exp (-\mathrm{i} l \varphi),
\end{gathered}
$$

where $z_{0}=k w_{0}^{2} / 2$ is the Rayleigh distance, $w_{0}$ is the beam waist of the fundamental or Gaussian mode $(n=0$,

\footnotetext{
* corresponding author; e-mail: suzana topuzoski@yahoo.com
}

$l=0), w(z)=w_{0}\left[1+\left(z / z_{0}\right)^{2}\right]^{1 / 2}$ is the beam transverse amplitude profile radius of the fundamental mode at distance $z$ from its beam waist, and $R(z)=z\left[1+\left(z_{0} / z\right)^{2}\right]$ is a real on-axial radius of curvature. $L_{n}^{l}\left(2 r^{2} / w^{2}(z)\right)$ is the generalized Laguerre polynomial of $n$-th order, and the coeficient

$$
C_{l, n}=\frac{2}{\sqrt{1+\delta_{0, l}}} \sqrt{\frac{n !}{\pi(l+n) !}} .
$$

The helical axicon is a hybrid of an axicon and a spiral phase plate. Its transmission function in the thin transparency approximation (approximating $\sin \gamma \approx \gamma$ ) is given by

$$
T(r, \varphi)=A(r) \exp (\mathrm{i} \alpha r-\mathrm{i} p \varphi),
$$

where the axicon parameter $\alpha=k(n-1) \gamma$ is connected to its refractive index $n$ for the incident beam of wavelength $\lambda$ and its internal angle (the angle on the axicon base) $\gamma$, and $k$ is wave number. The integer $p$ is the topological charge of the phase spiral plate, and also of the helical axicon (its sign defines the rotation direction of the phase layer, whereas its value shows how many times the phase is changed for a rate of $2 \pi$ in a $2 \pi$ cycle around the mode circumference).

The incident beam is entering orthogonally to the plane $\Delta(r, \varphi, \zeta)$, where the helical axicon is situated, a distance $z=\zeta$ from its beam waist, passing with its optical axis $z$ through the centre of the HA. At this distance the transverse amplitude profile radius of the LG beam is $[6]$ :

$$
\sigma_{n}^{l}(\zeta)=w(\zeta) \sqrt{2 n+l+1} .
$$

The function $A(r)$ in Eq. (2) is beam truncation function, when the helical axicon radius $R_{0}$ is smaller or equal to $\sigma_{n}^{l}(\zeta)$, defined as

$$
A(r)= \begin{cases}1, & \text { when } \beta>1, \\ \operatorname{circ}\left(r / \beta \sigma_{n}^{l}(\zeta)\right), & \text { when } \beta \leq 1\end{cases}
$$

with $\beta=R_{0} / \sigma_{n}^{l}(\zeta)$.

The intensity distribution of the incident beam, at distance $z=\zeta$, is 


$$
\begin{aligned}
& I(r, \varphi, \zeta)=\left|U_{n}^{l}(r, \varphi, \zeta)\right|^{2}=C_{l, n}^{2} \frac{w_{0}^{2}}{w^{2}(\zeta)}\left(\frac{2 r^{2}}{w^{2}(\zeta)}\right)^{l} \\
& \quad \times \exp \left(\frac{-2 r^{2}}{w^{2}(\zeta)}\right)\left[L_{n}^{l}\left(\frac{2 r^{2}}{w^{2}(\zeta)}\right)\right]^{2} \\
& \quad=C_{l, n}^{2}\left(w_{0}^{2} / w^{2}(\zeta)\right) I_{n}^{l}(r ; w(\zeta)) .
\end{aligned}
$$

\section{The diffracted wave field and intensity distribution}

In the observation screen $\Pi(\rho, \theta, z)$, situated at a distance $(z-\zeta)$ from the diffractive optical element plane, the wave field in the point $(\rho, \theta, z)$ is found using the Fresnel-Kirchhoff diffraction integral [7]:

$$
\begin{aligned}
& U(\rho, \theta, z)=\frac{\mathrm{i} k}{2 \pi(z-\zeta)} \\
& \quad \times \exp \left(-\mathrm{i} k\left((z-\zeta)+\frac{\rho^{2}}{2(z-\zeta)}\right)\right) \\
& \quad \times \iint_{\Delta} T(r, \varphi) U_{n}^{l}(r, \varphi, \zeta) \\
& \quad \times \exp \left(-i \frac{k}{2}\left(\frac{r^{2}}{z-\zeta}-\frac{2 r \rho \cos (\varphi-\theta)}{z-\zeta}\right)\right) r \mathrm{~d} r \mathrm{~d} \varphi,(6)
\end{aligned}
$$

where $\Delta$ is the area of the diffractive optical element which contributes to the diffraction. The integration over the azimuthal coordinate results into

$$
\begin{aligned}
& U(\rho, \theta, z)=C_{l, n} \frac{\mathrm{i} k}{(z-\zeta)} \frac{w_{0}}{w(\zeta)}\left(\frac{\sqrt{2}}{w(\zeta)}\right)^{l} \\
& \quad \times \exp (\mathrm{i}(l+p)(\theta+\pi / 2)) \times \exp (-\mathrm{i}(k z \\
& \left.\left.\quad+\frac{k \rho^{2}}{2(z-\zeta)}-(2 n+l+1) \arctan \left(\zeta / z_{0}\right)\right)\right) \int_{0}^{\infty} A(r) \\
& \quad \times J_{l+p}\left(\frac{k \rho r}{z-\zeta}\right) r^{l+1} L_{n}^{l}\left(\frac{2 r^{2}}{w^{2}(\zeta)}\right) \exp \left(\frac{-r^{2}}{w^{2}(\zeta)}\right) \\
& \quad \times \exp \left(-\mathrm{i} k\left(\frac{r^{2}}{2}\left(\frac{1}{z-\zeta}+\frac{1}{R(\zeta)}\right)-\alpha_{0} r\right)\right) \mathrm{d} r
\end{aligned}
$$

where we have denoted: $\alpha / k=\alpha_{0}=(n-1) \gamma$, while the integration over the radial coordinate has been performed using the stationary phase method [7]. The solution is evaluated around the stationary point

$$
r_{\mathrm{c}}=\frac{\alpha}{k} \frac{(z-\zeta) R(\zeta)}{[R(\zeta)+(z-\zeta)]} .
$$

Thus, the analytical expression for the diffracted wave field is derived in the form

$$
\begin{aligned}
& U(\rho, \theta, z)=\mathrm{i} C_{l, n}\left[\frac{w_{0}^{2} k \alpha_{0}}{w(\zeta) \sqrt{2}}\right]^{\frac{1}{2}} A\left(r_{\mathrm{c}}\right)\left(1+\frac{z-\zeta}{R(\zeta)}\right)^{-1} \\
& \quad \times \exp (-\mathrm{i} \phi) \exp \left(\mathrm{i}(l+p)\left(\theta+\frac{\pi}{2}\right)\right)
\end{aligned}
$$

$$
\begin{aligned}
& \times\left\{\frac{\sqrt{2}}{\left(w(\zeta) / \alpha_{0}\right)} \frac{(z-\zeta)}{[1+(z-\zeta) / R(\zeta)]}\right\}^{l+1 / 2} \\
& \times \exp \left(-\frac{(z-\zeta)^{2}}{\left(w(\zeta) / \alpha_{0}\right)^{2}(1+(z-\zeta) / R(\zeta))^{2}}\right) \\
& \times J_{l+p}\left(\frac{k \alpha_{0}}{1+(z-\zeta) / R(\zeta)} \rho\right) \\
& \times L_{n}^{l}\left(\frac{2(z-\zeta)^{2}}{\left(w(\zeta) / \alpha_{0}\right)^{2}(1+(z-\zeta) / R(\zeta))^{2}}\right),
\end{aligned}
$$

where the longitudinal phase $\phi$ is

$$
\begin{aligned}
\phi & =k\left[z+\frac{\rho^{2}}{2(z-\zeta)}-\frac{\alpha_{0}^{2}}{2} \frac{(z-\zeta)}{[1+(z-\zeta) / R(\zeta)]}\right. \\
& \left.-\frac{(2 n+l+1)}{k} \arctan \left(\frac{\zeta}{z_{0}}\right)\right] .
\end{aligned}
$$

The output Bessel type beam has a phase singularity along its optical axis $\rho=0$ and carries topological charge $l+p$, except for the case when $l$ and $p$ have equal values but opposite signs $(l-p=0)$. In longitudinal direction the beam is described by Laguerre-Gaussian function. It is diverging beam (the Bessel function argument depends on the $z$ coordinate).

As we have shown earlier in [4], for suitable choice of the incident beam parameters (when the HA is situated in the beam waist plane, where $\zeta=0$, $R(\zeta)=R(0) \rightarrow \infty)$, instead being described with Eq. (7), the output beam is obtained as a nondiverging Bessel beam and is defined as

$$
\begin{aligned}
& U(\rho, \theta, z)=\mathrm{i} C_{l, n}\left(\frac{w_{0} k \alpha_{0}}{\sqrt{2}}\right)^{1 / 2} A\left(r_{\mathrm{c}}^{\prime}\right)\left[\frac{\sqrt{2} z}{\left(w_{0} / \alpha_{0}\right)}\right]^{l+1 / 2} \\
& \quad \times \exp \left(-\mathrm{i} \phi^{\prime}\right) \exp (\mathrm{i}(l+p)(\theta+\pi / 2)) \\
& \times \exp \left(-\frac{z^{2}}{\left(w_{0} / \alpha_{0}\right)^{2}}\right) L_{n}^{l}\left(\frac{2 z^{2}}{\left(w_{0} / \alpha_{0}\right)^{2}}\right) J_{l+p}\left(k \alpha_{0} \rho\right),(8)
\end{aligned}
$$

where the longitudinal phase is

$$
\phi^{\prime}=k\left[z+\frac{\rho^{2}}{2 z}-\frac{\alpha_{0}^{2} z}{2}\right],
$$

while the critical point value is $r_{\mathrm{c}}^{\prime}=\alpha_{0} z$, and

$$
A\left(r_{\mathrm{c}}^{\prime}\right)= \begin{cases}1 ; & \text { when } R_{0} / \sigma_{n}^{l}(0)>1 \\ \operatorname{circ}\left(r_{\mathrm{c}}^{\prime} / R_{0}\right) ; & \text { when } R_{0} / \sigma_{n}^{l}(0) \leq 1\end{cases}
$$

The intensity distribution of the beam (8) is

$$
\begin{aligned}
& I(\rho, \theta, z)=C_{l, n}^{2}\left(\frac{w_{0} k \alpha_{0}}{\sqrt{2}}\right) A\left(r_{\mathrm{c}}^{\prime}\right)\left[\frac{2 z^{2}}{\left(w_{0} / \alpha_{0}\right)^{2}}\right]^{l+\frac{1}{2}} \\
& \quad \times \exp \left(\frac{-2 z^{2}}{\left(w_{0} / \alpha_{0}\right)^{2}}\right)\left[L_{n}^{l}\left(\frac{2 z^{2}}{\left(w_{0} / \alpha_{0}\right)^{2}}\right)\right]^{2} \\
& \quad \times J_{l+p}^{2}\left(k \alpha_{0} \rho\right),
\end{aligned}
$$

or, it can be, also, written as 


$$
\begin{aligned}
& I(\rho, \theta, z)=C_{l, n}^{2}\left(w_{0} k \alpha_{0} / \sqrt{2}\right) A\left(r_{\mathrm{c}}^{\prime}\right) I_{n}^{l+1 / 2}\left(z ; w_{0} / \alpha_{0}\right) \\
& \quad \times J_{l+p}^{2}\left(k \alpha_{0} \rho\right) .
\end{aligned}
$$

The vortex radius of the nondiverging Bessel beam (9) retains its value unchanged within the propagation interval. It can be calculated as $\rho_{\max }=\mu_{l+p, 1} / k \alpha_{0}$, where $\mu_{l+p, 1}$ is found as a root of the equation: $\left|J_{|l+p|+1}\left(k \alpha_{0} \rho\right)\right|=$ $\left|J_{|l+p|-1}\left(k \alpha_{0} \rho\right)\right|$.

When $\beta \leq 1$, the function $A\left(r_{\mathrm{c}}^{\prime}\right)$ dictates the longitudinal truncation of the outgoing Bessel beam. The beam has maximum propagation distance

$$
L_{\max }=\beta \sigma_{n}^{l}(0) / \alpha_{0}=R_{0} / \alpha_{0} .
$$

The function

$$
\begin{aligned}
& I_{n}^{l+1 / 2}\left(z ; w_{0} / \alpha_{0}\right)=\left[\frac{2 z^{2}}{\left(w_{0} / \alpha_{0}\right)^{2}}\right]^{l+1 / 2} \exp \left(\frac{-2 z^{2}}{\left(w_{0} / \alpha_{0}\right)^{2}}\right) \\
& \quad \times\left[L_{n}^{l}\left(\frac{2 z^{2}}{\left(w_{0} / \alpha_{0}\right)^{2}}\right)\right]^{2}
\end{aligned}
$$

in Eq. (10), being of Laguerre-Gaussian type, is similar to that one in the expression (5); the difference is that it is related to the axial variable $z$ instead to the radial variable $r$, and it has half integer power in its definition. When $\beta>1$ the beam ranging distance is

$$
\sigma_{n}^{l+1 / 2}=\left(w_{0} / \alpha_{0}\right) \sqrt{2 n+l+3 / 2} .
$$

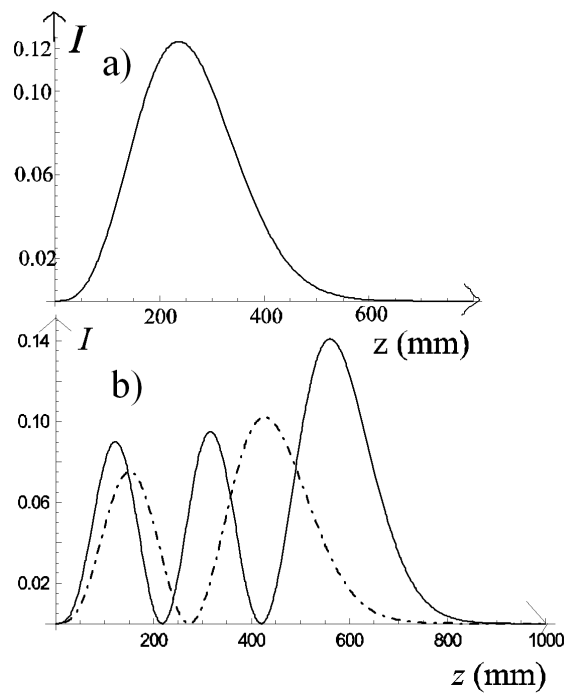

Fig. 1. The intensity variation along $z$ axis for: (a) $p=1, n=0, l=1$, (b) $p=1, n=1, l=1$ (dotted-dashed curve), $p=1, n=2, l=1$ (solid curve).

The inhomogeneity of the intensity along longitudinal direction can be seen in Fig. 1, where the intensity has been calculated in the first bright ring, at position $\rho_{\max }$, based on Eq. (9), when $A\left(r_{\mathrm{c}}^{\prime}\right)=1$, for different values of $p, n$ and $l$. The used parameters for the axicon and the incident beam are: $\gamma=1.35^{\circ}, n=1.48$ for wavelength $\lambda=980 \mathrm{~nm}, w_{0}=3 \mathrm{~mm}$. Along the longitudinal di-

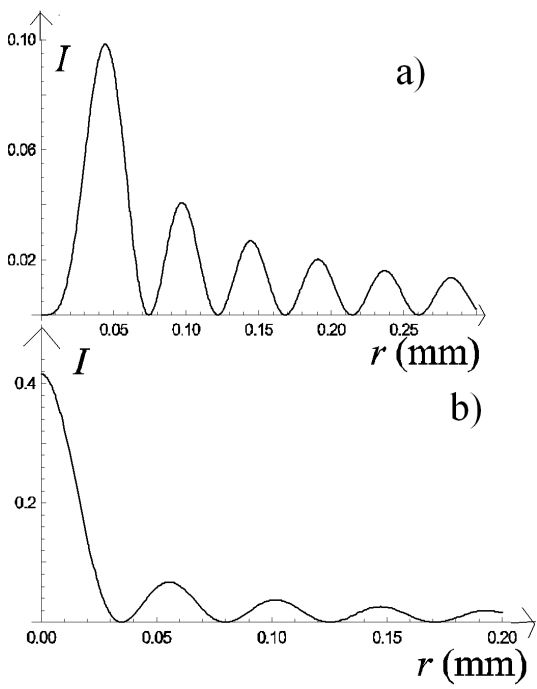

Fig. 2. Radial intensity distribution at distance $z=450 \mathrm{~mm}$ for: (a) $p=1, n=1, l=1$, (b) $p=-1$, $n=1, l=1$.

rection the intensity oscillates, reaching maximum values $(n+1)$ times (similarly to the radial intensity distribution of the incident beam). The intensity interceptions with zero-values points along the $z$-coordinate, and the beam nondiverging feature could be interesting for particles optical trapping in few spatially separated chambers [1].

In Fig. 2 we have shown the radial intensity distribution when $A\left(r_{\mathrm{c}}^{\prime}\right)=1$ : (a) when $p=1, l=1, n=1$, the output beam is vortex, while when the topological charges of the HA and the incident beam are equal in modulus but with opposite signs $(p=-l)$, the Bessel beam is chargeless (b). When being specialized for $n=0$ the results get the forms as in [4].

\section{Conclusion}

We have derived the analytical expression for the diffracted wave field when LG beam of phase singularity $l$ and radial mode number $n$ undergoes diffraction by helical axicon. Further, the propagation distance and the vortex radius of the output beam have been discussed.

\section{References}

[1] D. McGloin, K. Dholakia, Contemp. Phys. 46, 15 (2005).

[2] J. Durnin, J.J. Miceli, J.H. Eberly, Phys. Rev. Lett. 58, 1499 (1987).

[3] J. Arlt, K. Dholakia, Opt. Commun. 177, 297 (2000).

[4] S. Topuzoski, Lj. Janicijevic, Opt. Commun. 282, 3426 (2009).

[5] H. Kogelnik, T. Li, Appl. Opt. 5, 1550 (1966).

[6] R.L. Phillips, L.C. Andrews, Appl. Opt. 22, 643 (1983).

[7] M. Born, E. Wolf, Principles of Optics, Univ. Press, Cambridge 1999. 